\title{
Fitting fatigue data with a bi-conditional model
}

\author{
$M$ COVA $^{1,2}$ and R TOVO ${ }^{1}$ \\ ${ }^{1}$ Department of Engineering, University of Ferrara, v. Saragat 1, 44100 Ferrara, Italy, ${ }^{2}$ SACMI Imola S.C., v. Selice 17/A, 40026 Imola, Italy \\ Received Date: 15 March 2016; Accepted Date: 24 September 2016; Published Online:
}

A B S TRACT The formulation of a probability-stress-life (P-S-N) curve is a necessary step beyond the basic S-N relation when dealing with reliability. This paper presents a model, relevant to materials that exhibits a fatigue limit, which considers the number of cycles to failure and the occurrence of the failure itself as statistically independent events, described with different distributions and/or different degree of scatter. Combining these two as a parallel system leads to the proposed model. In the case where the S-N relation is a Basquin's law, the formulations of the probability density function, cumulative distribution function, quantiles, parameter and quantile confidence interval are presented in a procedure that allows practically any testing strategy.

The result is a flexible model combined with the tools that deliver a wide range of information needed in the design phase. Finally, an extension to include static strength and applicability to fatigue crack growth and defects-based fatigue approach are presented.

Keywords bootstrap; confidence interval; high cycle fatigue; probabilistic analysis; reliability analysis; S-N curves.

NOMENCLATURE
$A=$ constant in the Basquin's law

$a=$ characteristic size of the material discontinuity

$b=$ slope in the Basquin's law

$\mathrm{C}=$ constant in the Paris-Erdogan relation

$\Delta K=$ SIF range

$\Delta K_{t h}=$ threshold SIF range

$F^{e n d}=$ probability of endurance

$F^{e x i}=$ probability of existence

$F^{s t}=$ probability of static strength

$K_{I C}=$ plain strain fracture toughness

$L=$ likelihood function

$\mathscr{L}=\log$-likelihood function

$N=$ number of cycles

$N_{f}=$ number of cycles to failure

= natural logarithm

$\tilde{N}$ = array of all the $N_{i}$ from the real test greater or equal of the minimum run out

$N_{j}^{v}=$ virtual number of cycles corresponding to the applied stress and the (randomly) chosen probability

$N^{R-O}=$ array of number of cycles of the run-out (not broken) specimens

$n=$ exponent in the Paris-Erdogan relation

$P=$ probability of failure

$\boldsymbol{P}_{j}=$ vector of probability values, randomly chosen at the $\mathrm{j}$-th iteration of the bootstrap procedure

$\mathbb{Q}=$ quantile 


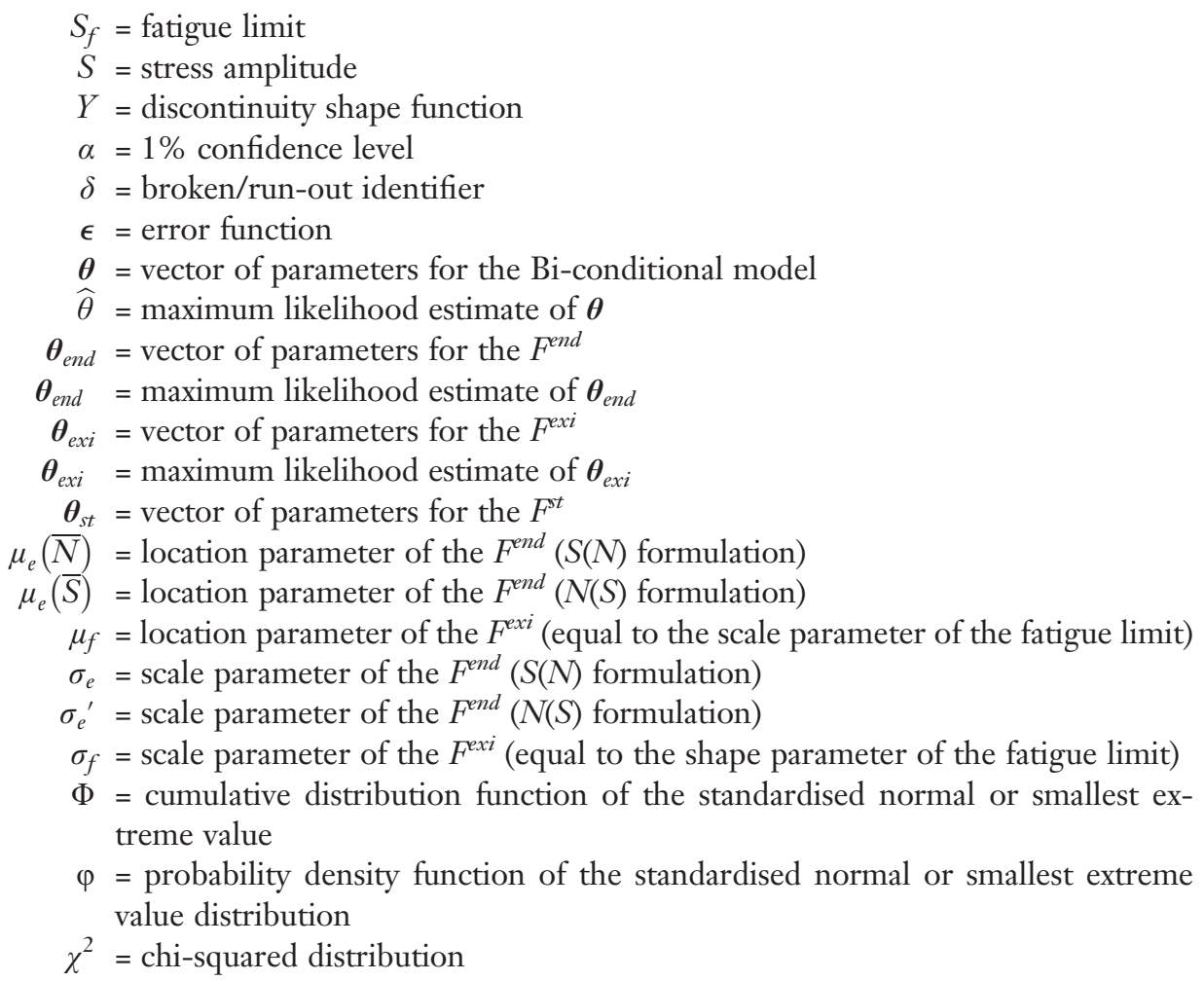

\section{INTRODUCTION}

The outcome of a fatigue test has usually at least these two features:

- Fatigue life is inversely dependent from the applied stress cycle amplitude; and

- Fatigue life exhibits some degree of scatter.

In its simplest form, $\log (\mathrm{N})$ is a linear function of $\log$ (S) ${ }^{1-3}$ In special cases, more complex behaviour may be observed $^{4}$. However, for a class of materials and conditions (e.g. steels and cast irons with $\mathrm{N}$ up to $10^{8}$ ) of wide use, fatigue tests outcome exhibits a change in slope associated to the so-called knee point.

After that point, it is still disputed whether the following trend has a lower slope ${ }^{5,6}$ or leads to an horizontal asymptote. In the latter case, the asymptote value is also known as the fatigue limit, that is a stress level below which further cycling does not lead to failure. This paper deals with this latter family of fatigue curves (Fig. 1).

Although the statistical nature of fatigue data has been widely acknowledged from the very beginning, fatigue data or properties are often presented in the form of median S-N curves, for either the finite life region or the fatigue limit. On the other hand, the designer needs to take into account an appropriate safety margin, because no production can withstand a $50 \%$ failure rate. Such safety margin can be somehow implicit in the classical 'safety factor approach', but being able to express a probability of failure is a much more powerful tool.

Components subjected to fatigue with a design endurance over $10^{7}$ and a feasible failure probability below $0.1 \%$ are the focus of this research. In essence, what is needed at the end is the fatigue strength distribution at the target design endurance. Together with testing strategy, data analysis is crucial for deriving this information with confidence.

The stair-case strategy is the most oriented on the fatigue limit. However, either in its original Dixon and Mood's form ${ }^{7}$ or in more general Maximum Likelihood formulations, ${ }^{8}$ the data analysis is strictly based on a quantal approach, and thus, each test outcome is merely a 'broken' or a 'run out'. By doing so, an important information such as the number of cycles to failure remains unused. Consequently, although recent efforts $^{9-11}$ have tried to optimise this methodology, its ability to describe the scatter with sufficient confidence remains limited. ${ }^{12}$

In order to take the number of cycles to failure into account, a full P-S-N relationship must be employed, and in recent years, a renewed interest in such field has led to various models. However, many models like the Random Fatigue Limit Model ${ }^{13}$ or the one proposed by Castillo and Fernandez-Canteli, ${ }^{14}$ although built on strong statistical basis, tie a fairly strong bond between the High (sometimes the Low) and Very High Cycle 


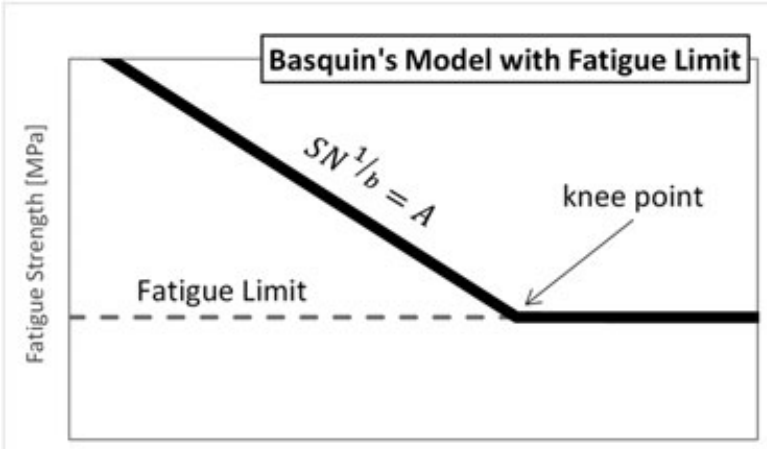

Cycles to Failure

Fig. 1 Basquin's law with fatigue limit.

Fatigue behaviour, implicitly assuming similarities in the probabilistic outcome of different damaging mechanisms. A more disjointed approach is proposed by Loren $\mathrm{et} \mathrm{al} .{ }^{15}$ and Paolino et al., ${ }^{16,17}$ whose models allow for a different degree of scatter in strength between the finite life region and the fatigue limit.

The model proposed in this paper reinterprets a well-known and vastly used basic representation under a probabilistic fashion. Although independently developed, it is heavily linked to those of Pascual and Meeker and Loren, and the resulting cumulative distribution function (CDF) is formally a subcase of the one proposed by Paolino et al. Its originality lies in the interpretation of the underlying damaging mechanisms and in the detailed presentation of all the tools and procedures needed for a full practical implementation, including the confidence interval estimate for the parameters and the quantiles.

Finally, extensions to other uses are presented.

\section{BI-CONDITIONAL MODEL: GENERAL CONCEPTS}

Within an S-N model with fatigue limit, let us consider that there are two distinct and independent zones, separated by the fatigue limit. A relation between the stress amplitude and the cycles to failure is present only in the first part, and it will be referred to as the endurance relation. Moving to a statistical model, this relation is thus independent from the limiting condition (i.e. the fatigue limit) as long as the number of cycles at the knee point is not defined a priori. The two models are hence statistically independent. This hypothesis is often adopted in the testing analysis or in the design schematization of the fatigue strength curves.

Let us consider the cycles to failure when the $S$ stress is cyclically applied $N_{f}(S)$ a random variable. Then we define the probability of endurance as the marginal probability that the fatigue life $N_{f}(S)$ at an applied (fixed) stress $S$ is less than the performed number of cycles $N$. That is as follows:

$F_{N_{f} \mid S}^{\text {end }}=\operatorname{Pr}\left(N_{f}(S) \leq N\right)=F^{\text {end }}\left(N, S, \boldsymbol{\theta}_{\text {end }}\right)$

where $\boldsymbol{\theta}_{\boldsymbol{e n d}}$ is an adequate set of parameters.

If we also consider the fatigue limit as random variable, we consecutively define the probability of the existence of the failure as

$F_{S_{f}}^{e x i}=\operatorname{Pr}\left(S_{f} \leq S\right)=F^{e x i}\left(S, \boldsymbol{\theta}_{e x i}\right)$

where $S_{f}$ is the fatigue limit. $\boldsymbol{\theta}_{\boldsymbol{e x i}}$ is a convenient set of parameters, and, in general, it is different from $\boldsymbol{\theta}_{\text {end }}$ not only because the formulations of $F^{e n d}$ and $F^{e x i}$ are different but also because the scatter is different.

If the two events, meaning the number of cycles to failure and the happening of the failure itself, are considered as independent, then the overall behaviour can be considered as parallel system in which both conditions must be satisfied for the failure to happen (Fig. 2). That is, to have failure before $N$, the specimen/component must be loaded at a stress level $S$ above its fatigue limit and $N_{f}$ at $S$ must be lower than $N$.

From the theory of parallel systems, it then follows that the overall probability of failure is the product of the individual probabilities

Probability of failure $=F_{N_{f} \mid S}^{e n d} \cdot F_{S_{f}}^{e x i}$

The concept of two independent conditions, which both need to be satisfied for failure to happen, is the core of the model and falls in the 'Dominant role model' class in Harlow's overview of bimodal behaviours. ${ }^{18}$ A similar approach can be found also in Beretta et al. ${ }^{19}$ in dealing with extreme value distributions of multiple defect populations, although they do not explicitly refer to a parallel system, and they call it 'Competing risk model' (which is the name Harlow uses for the series system model).

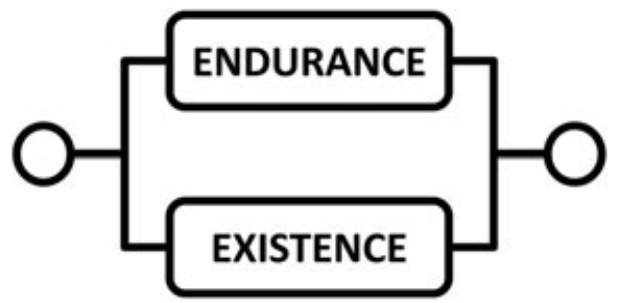

Fig. 2 Fatigue failure modelled as a parallel system. 


\section{BI-CONDITIONAL: ANALYTICAL FORMULATION}

For the finite life region of the Wohler curve, the wellknown Basquin relation ${ }^{3}$ offers a convenient representation, which implies a linear relationship between the applied stress $\mathrm{S}$ and the resulting life $N_{f}$ in a $\log -\log$ diagram.

$S N_{f}^{1 / b=A}$

If we treat $N_{f}$ as random variable (as in Grove et al. ${ }^{10}$ ) and by working with natural logarithms, we can use the Basquin equation to model the cycles to failure:

$\ln N_{f}=b(\ln A-\ln S)+\epsilon$

where $\epsilon$ is the error term.

By indicating the natural logarithm with the accent ${ }^{-}$, we assume the marginal CDF of the endurance as (Fig. 4a)

$$
\begin{aligned}
F_{\overline{N_{f}} \mid \bar{S}}^{e n d} & =\operatorname{Pr}\left(\overline{N_{f}}(\bar{S}) \leq \bar{N}\right)=F^{\text {end }}\left(\bar{N}, \bar{S}, \bar{A}, b, \sigma_{e}\right) \\
& =\Phi\left(\frac{\bar{N}-b(\bar{A}-\bar{S})}{b \sigma_{e}}\right)=\Phi\left(\frac{\bar{N}-\mu_{e}(\bar{S})}{\sigma_{e}{ }^{\prime}}\right)
\end{aligned}
$$

where $\mu_{e}(\bar{S})=b(\bar{A}-\bar{S})$ is the location parameter; $\sigma_{e}$ is the scale parameter of the distribution of $S$ at given $N$; $\sigma_{e}{ }^{\prime}=b \sigma_{e}$ is the scale parameter of $N$ at given $S$; and $\Phi$ is either the standardised normal $(\mathcal{N})$ or the smallest extreme value (SEV) distribution. It is worth noting that if $\ln (\mathrm{x})$ follows a SEV distribution, then $\mathrm{x}$ is Weibull-distributed.
With the given formulation, the iso-probability curves are ( $P$ is the probability) as follows:

$\overline{N_{f}}=b(\bar{A}-\bar{S})+\sigma_{e}^{\prime} \cdot \Phi^{-1}(P)$

and hence, they are a bundle of lines with common slope (-b) and intercept $\left(b \bar{A}+\sigma_{e}{ }^{\prime} \cdot \Phi^{-1}(P)\right)$ in an $\bar{N} v s \bar{S}$ diagram (Fig. 3).

The marginal probability density function (PDF) of $\overline{N_{f}}$ is (Fig. 4b)

$$
\begin{aligned}
& f_{\bar{N}_{f} \mid \bar{S}}^{\text {end }}\left(\bar{N}, \bar{S}, \bar{A}, b, \sigma_{e}\right)=f_{\overline{N_{f}} \mid \bar{S}}^{\text {end }}\left(\bar{N}, \bar{S}, \boldsymbol{\theta}_{\text {end }}\right)=\frac{\partial F^{\text {end }}}{\partial \bar{N}}= \\
& =\frac{\partial\left(\frac{\bar{N}-\mu_{e}(\bar{S})}{\sigma_{e}{ }^{\prime}}\right)}{\partial \bar{N}} \varphi\left(\frac{\bar{N}-\mu_{e}(\bar{S})}{\sigma_{e}{ }^{\prime}}\right)=\frac{1}{\sigma_{e}{ }^{\prime}} \varphi\left(\frac{\bar{N}-\mu_{e}(\bar{S})}{\sigma_{e}{ }^{\prime}}\right)
\end{aligned}
$$

where $\varphi$ is the PDF of the standard normal or of the SEV.

If the distribution of the fatigue strength at given number of cycles $\bar{S}_{e}(\bar{N})$ is of interest, the formulation can be reversed as follows:

$$
\begin{aligned}
F_{\overline{S_{e}} \mid \bar{N}}^{\text {end }} & =\operatorname{Pr}\left(\bar{S}_{e}(\bar{N}) \leq S\right)=\Phi\left(\frac{\bar{N}-b(\ln A-\bar{S})}{\sigma_{e}{ }^{\prime}}\right) \\
& =\Phi\left(\bar{S}-\left(\frac{\left.\ln A-\frac{\bar{N}}{b}\right)}{\sigma_{e}{ }^{\prime} / b}\right)=\Phi\left(\frac{\bar{S}-\mu_{e}(\bar{N})}{\sigma_{e}}\right)\right.
\end{aligned}
$$

Similar to the previous case, at any given value of $\bar{N}, \overline{S_{e}}$ is distributed with parameters $\left[\ln A-\frac{\bar{N}}{b ; \sigma_{e}}\right]$.

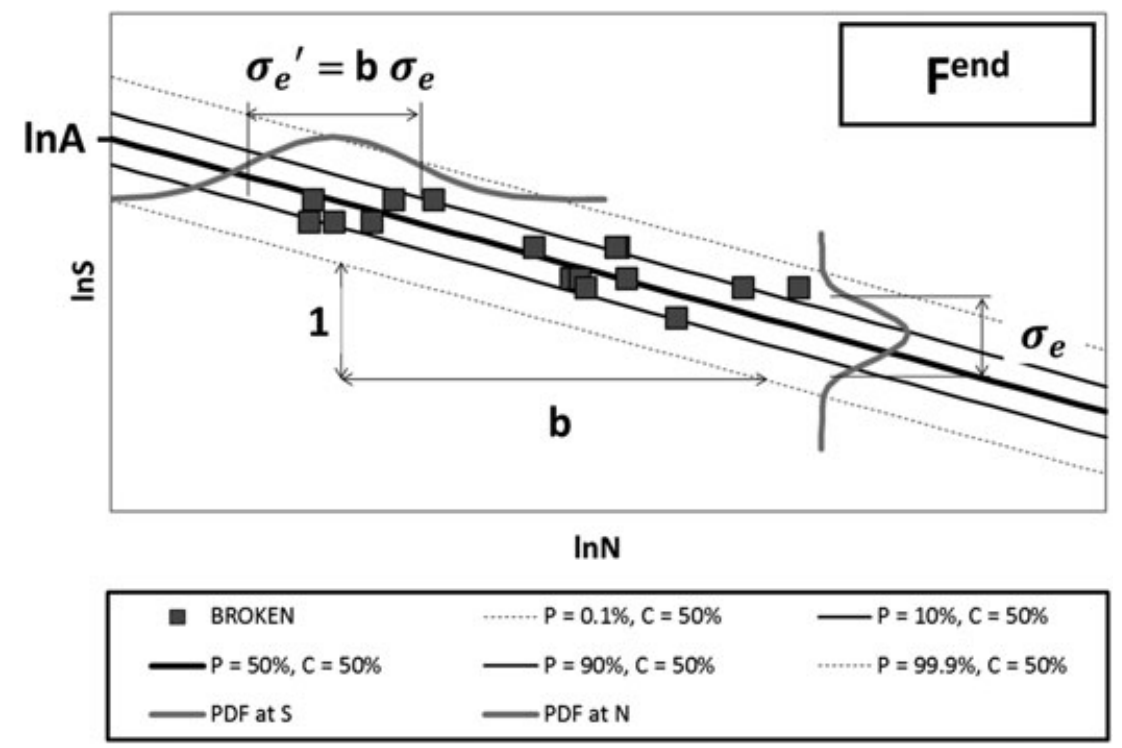

Fig. 3 Graphical representation of the $F^{\text {end }}$ parameters. 
a)

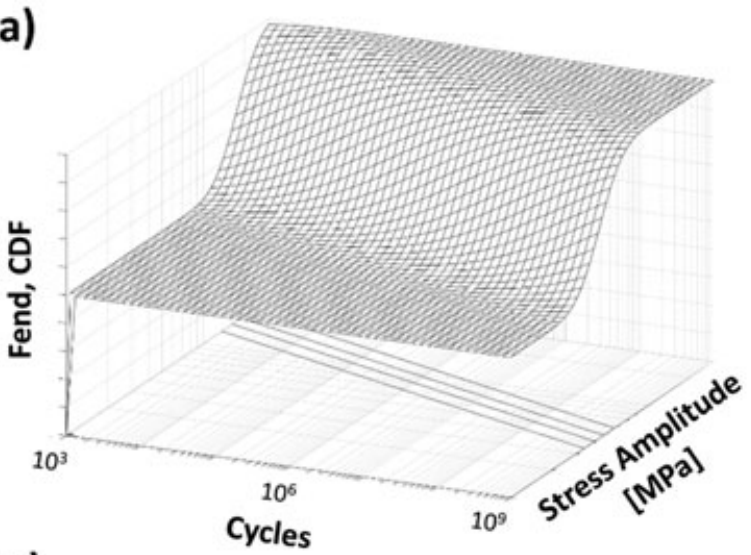

c)

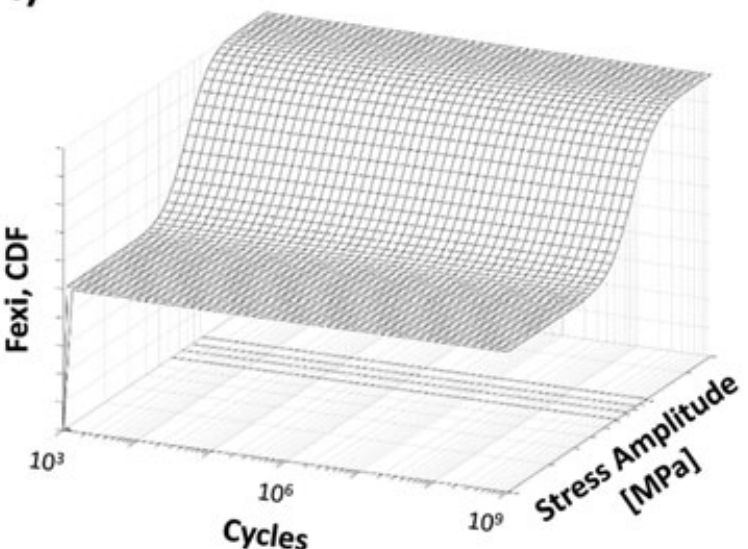

e)

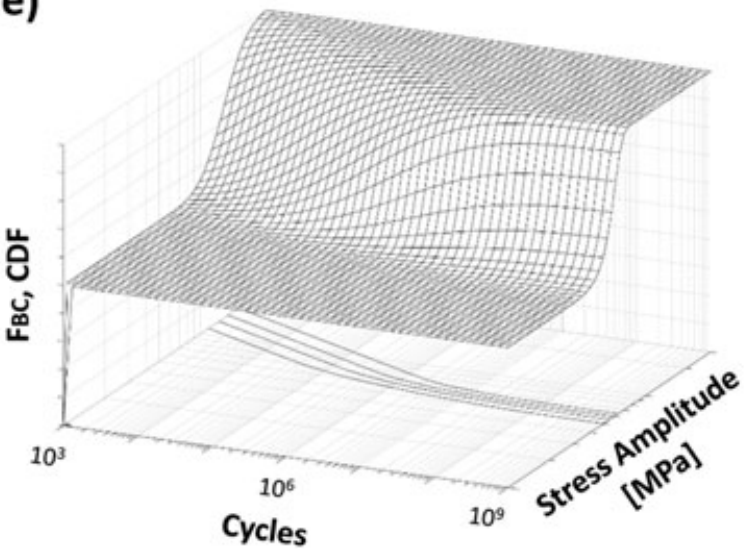

b)

d)
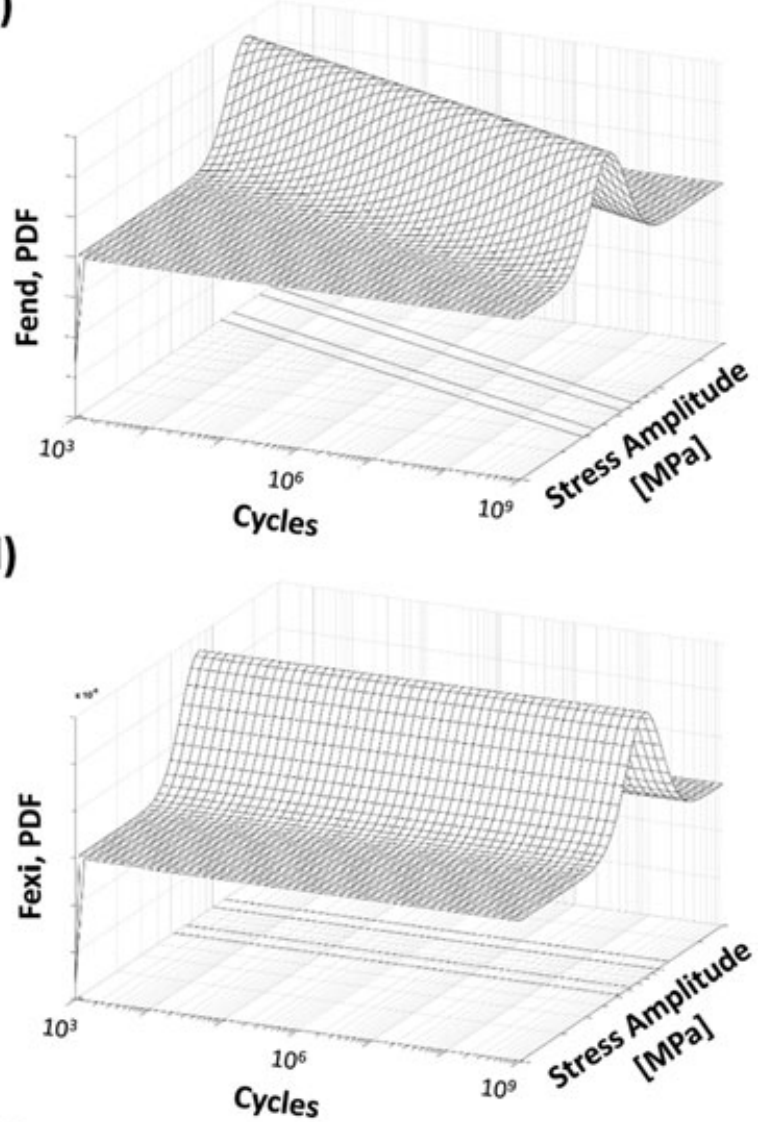

f)

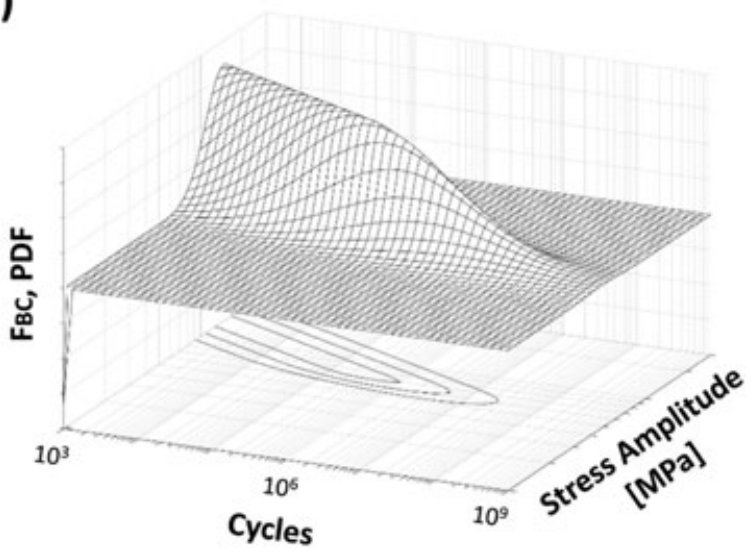

Fig. 4 (a) Cumulative distribution function of $\mathrm{F}^{\mathrm{end}}$ (6); (b) Marginal PDF of $\mathrm{F}^{\mathrm{end}}$ (8); (c) Cumulative distribution function of $\mathrm{F}^{\mathrm{exi}}$ (11); (d) Probability density function of $\mathrm{F}^{\mathrm{exi}}$ (11); (e) Cumulative distribution function of the BCM (27); (f) Marginal PDF of the BCM (14).

In analogy with what have been shown before, the marginal PDF of $\overline{S_{e}}$ is as follows:
In many engineering applications, the distribution in strength at given $\mathrm{N}$ (i.e. $\left.F_{\overline{S_{e}} \mid \bar{N}}\right)$ is more useful than the dis-

$f_{\overline{S_{e}} \mid \bar{N}}^{\text {end }}\left(\bar{N}, \bar{S}, A, b, \sigma_{e}\right)=f_{\overline{S_{e}} \mid \bar{N}}^{\text {end }}\left(\bar{N}, \bar{S}, \boldsymbol{\theta}_{\text {end }}\right)=\frac{\partial F_{\overline{S_{e}} \mid \bar{N}}^{\text {end }}}{\partial \bar{S}}=\frac{\partial\left(\frac{\bar{S}-\mu_{e}(\bar{N})}{\sigma_{e}}\right)}{\partial \bar{S}} \varphi\left(\frac{\bar{S}-\mu_{e}(\bar{N})}{\sigma_{e}}\right)=\frac{1}{\sigma_{e}} \varphi\left(\frac{\bar{S}-\mu_{e}(\bar{N})}{\sigma_{e}}\right)$ 
(i.e. the target product life) and $\mathrm{S}$ is a design variable. On the other side, in fatigue testing, $S$ is the controlled (independent) variable, and $\mathrm{N}$ is the outcome of the test itself; that is why $F_{\overline{N_{f}}}^{\text {end }} \mid \bar{S}$ is the proper way for at least the fitting of the models to the data. In the standard, nonprobabilistic Basquin, the suggested approach is in fact the linear regression of $\bar{N}(\bar{S}) .{ }^{20}$

The $F_{\overline{N_{f}}}^{\text {end }} \mid \bar{S}$ option is hence chosen as the standard option for the model. However, to give a direct feeling of the distribution in $F_{\overline{S_{e}}}^{\text {end }} \mid \bar{N}$, the parameters are those of the latter.

The $F^{e x i}$ is the CDF of the fatigue limit, and hence, it is not a function of the number of cycles, that is in the proposed model, there is no slope in the iso-probability curve for the Very High Cycle Fatigue region. Its formulation is (Fig. 4c)

$F_{\overline{S_{f}}}^{e x i}=\operatorname{Pr}\left(\overline{S_{f}} \leq \bar{S}\right)=F^{e x i}\left(\bar{S}, \boldsymbol{\theta}_{\text {exi }}\right)=\Phi\left(\frac{\bar{S}-\mu_{f}}{\sigma_{f}}\right)$

with the same notation, $\Phi$ is again either the standard or the SEV CDF, $\mu_{f}$ is the location parameter (i.e. the characteristic value) and $\sigma_{f}$ is the scale parameter (i.e. the scatter parameter) of the fatigue limit.

The marginal PDF of S is simply (Fig. 4d)

$$
\begin{aligned}
f_{\overline{S_{f}}}^{e x i}\left(\bar{S}, \mu_{f}, \sigma_{f}\right) & =f_{\overline{S_{f}}}\left(\bar{S}, \boldsymbol{\theta}_{\text {exi }}\right)=\frac{\partial F^{e x i}}{\partial \bar{S}} \\
& =\frac{\partial\left(\frac{\bar{S}-\mu_{f}}{\sigma_{f}}\right)}{\partial \bar{S}} \varphi\left(\frac{\bar{S}-\mu_{f}}{\sigma_{f}}\right) \\
& =\frac{1}{\sigma_{f}} \varphi\left(\frac{\bar{S}-\mu_{f}}{\sigma_{f}}\right)
\end{aligned}
$$

Finally, the marginal CDF of the BCM is the product of $F^{\text {end }}$ and $F^{e x i}$ (Fig. 4e):

$$
\begin{aligned}
F_{\overline{N_{f}}} & =F\left(\bar{N}, \bar{S}, \bar{A}, b, \sigma_{e}, \mu_{f}, \sigma_{f}\right)=F^{B C}(\bar{N}, \bar{S}, \boldsymbol{\theta}) \\
& =\Phi\left(\frac{\bar{N}-\mu_{e}(\bar{S})}{\sigma_{e}^{\prime}}\right) \cdot \Phi\left(\frac{\bar{S}-\mu_{f}}{\sigma_{f}}\right)
\end{aligned}
$$

Again, $\Phi$ is either the normal or the SEV CDF, and, for the sake of clarity, the choice can be different for $F^{\text {end }}$ and $F^{e x i}$, leading to four combinations. Although it is the result of different assumptions and framework, this resulting CDF is very similar to the one presented in Loren et al. ${ }^{15}$ (see also Loren ${ }^{21}$ ) and to the case of 'one failure mode due to one cause with fatigue limit' in Paolino et al. ${ }^{16}$
The marginal PDF of the $\mathrm{BCM}$ is the partial derivative of $F_{\overline{N_{f}}}$ with respect to $\bar{N}$ (Fig. 4f):

$$
\begin{aligned}
& f_{\overline{N_{f}}}\left(\bar{N}, \bar{S}, \bar{A}, b, \sigma_{e}, \mu_{f}, \sigma_{f}\right)=f_{\overline{N_{f}}}(\bar{N}, \bar{S}, \boldsymbol{\theta})=\frac{\partial F_{\overline{N_{f}}}}{\partial \bar{N}} \\
& =\frac{\partial F^{e n d}}{\partial \bar{N}} \cdot \varphi\left(\frac{\bar{N}-\mu_{e}(\bar{S})}{\sigma_{e}^{\prime}}\right) \cdot F^{e x i}+F^{e n d} \cdot \frac{\overbrace{\frac{\partial F^{e x i}}{\partial \bar{N}}}^{=0} \cdot \varphi\left(\frac{\bar{S}-\mu_{f}}{\sigma_{f}}\right)}{=\frac{1}{\sigma_{e}^{\prime}} \cdot \varphi\left(\frac{\bar{N}-b(\bar{A}-\bar{S})}{\sigma_{e}^{\prime}}\right) \cdot \Phi\left(\frac{\bar{S}-\mu_{f}}{\sigma_{f}}\right)}
\end{aligned}
$$

where $\varphi$ is the PDF of the normal or the SEV.

The $P$ quantile at given $\bar{S}$ can be explicitly derived as follows:

$$
\begin{aligned}
& P=F^{B C}(\bar{N}, \bar{S}, \boldsymbol{\theta})=\Phi\left(\frac{\bar{N}-\mu_{e}(\bar{S})}{\sigma_{e}^{\prime}}\right) \cdot \Phi\left(\frac{\bar{S}-\mu_{f}}{\sigma_{f}}\right) \Rightarrow \\
& \bar{N}(P, \bar{S})=\mathbb{Q}(P, \bar{S})=\left\{\begin{array}{c}
\mu_{e}(\bar{S})+\sigma_{e}^{\prime} \Phi^{-1}\left(\frac{P}{\Phi\left(\frac{\bar{S}-\mu_{f}}{\sigma_{f}}\right)}\right) \\
\text { if } \bar{S}>\mu_{f}+\sigma_{f} \Phi^{-1}(P) \\
+\infty \quad \text { if } \bar{S} \leq \mu_{f}+\sigma_{f} \Phi^{-1}(P)
\end{array}\right.
\end{aligned}
$$

Unfortunately, there is no explicit formulation of the quantile at given $\mathrm{N}$, which is often of interest.

$$
\begin{gathered}
P=F^{B C}(\bar{N}, \bar{S}, \boldsymbol{\theta})=\Phi\left(\frac{\bar{S}-\mu_{e}(\bar{N})}{\sigma_{e}}\right) \cdot \Phi\left(\frac{\bar{S}-\mu_{f}}{\sigma_{f}}\right) \Rightarrow \\
\bar{S}(P, \bar{N})=\mu_{e}(\bar{N})+\sigma_{e} \Phi^{-1}\left(\frac{P}{\Phi\left(\frac{\bar{S}-\mu_{f}}{\sigma_{f}}\right)}\right)
\end{gathered}
$$

However, it can be found numerically by iterating this loop:

$$
\bar{S}_{i+1}(P, \bar{N})=\mu_{e}(\bar{N})+\sigma_{e} \Phi^{-1}\left(\frac{P}{\Phi\left(\frac{\bar{S}_{i}-\mu_{f}}{\sigma_{f}}\right)}\right) ;
$$

stop when $\left|\bar{S}_{i+1}-\bar{S}_{i}\right|<$ admissible error 
With this formulation, the iso-probability curves look as in Fig. 5. Some features are worth noting:

a) Two linear parts, which may be called HCF and VHCF areas, are smoothly connected by a curved transition zone;

b) These two parts are dominated, respectively, by $F^{\text {end }}$ and $F^{e x i}$. This also means that data within these regions will only influence the fitting of the dominating distribution. Data in the transition zone will influence both distributions (Fig. 6).

c) The HCF and VHCF areas can have, generally, different strength distributions and different scatter. This in fact was one of the characteristic hypothesis;

d) The transition, curved zone of each single isoprobability curve is not explicitly written in the model but is a result of the product of $F^{e n d}$ and $F^{e x i}$.

e) The transition region is practically the only one in which the model is 'truly effective', meaning $F^{\text {end }}$ and $F^{e x i}$ are both active; on the other hand, this is also the region with usually the highest density of experimental data;

f) The HCF zone has a constant degree of scatter, that is it assumes a homoscedastic behaviour. A heteroscedastic extension can be considered by adding a further parameter (see par 6.5).

\section{PARAMETER ESTIMATE PROCEDURE}

As it is stated in Eq. (13), with this formulation, this model has five parameters, whose fitting inevitably need an optimization through numerical tools. The consolidated tool for this kind of problem is the optimization of the parameters in order to maximise the model log-likelihood. With five (or more, if extensions are added up) parameters, this optimization is not trivial, and one crucial issue is the initial estimate (i.e. starting point for the optimization) of the parameters.

The suggested procedure is to separately perform a first estimate for the $F^{e x i}$ and $F^{\text {end }}$ parameters, then to adjust by considering the full $F^{B C}$. In the following, a step by step procedure is presented.

\section{First estimate of the $F^{e n d}$ parameters: A, b, $\sigma_{e}$}

The broken specimens usually produce the points $\left(\bar{N}_{i}, \bar{S}_{i}\right)$ that are mostly aligned following the Basquin relation [linear trend with some degree of scatter, see (5)]. Let us thus first consider only the broken specimens; a preliminary estimate of $\mathrm{A}$ and $\mathrm{b}$ can be drawn by a least square fitting of a linear interpolation of $\ln (\mathrm{N})$ versus $\ln$ (S). After that, one can make advantage of the homoscedasticity and project the $\left(\bar{N}_{i}, \bar{S}_{i}\right)$ points to an arbitrary value of stress amplitude $\bar{S}^{0}$. The array of resulting cycles to failure is composed of

$\bar{N}_{i}^{0}=\bar{N}_{i}\left(\bar{S}^{0}\right)=b\left(\bar{S}_{i}-\bar{S}^{0}\right)+\bar{N}_{i}$

This array can be interpreted as a univariate sample with 0.5 quantile equal to the intercept of the least square fitted line with the value $\bar{S}^{0}$. The scale parameter of the fitted distribution (either normal or SEV) can be taken as a first estimate of $\sigma_{e}$.

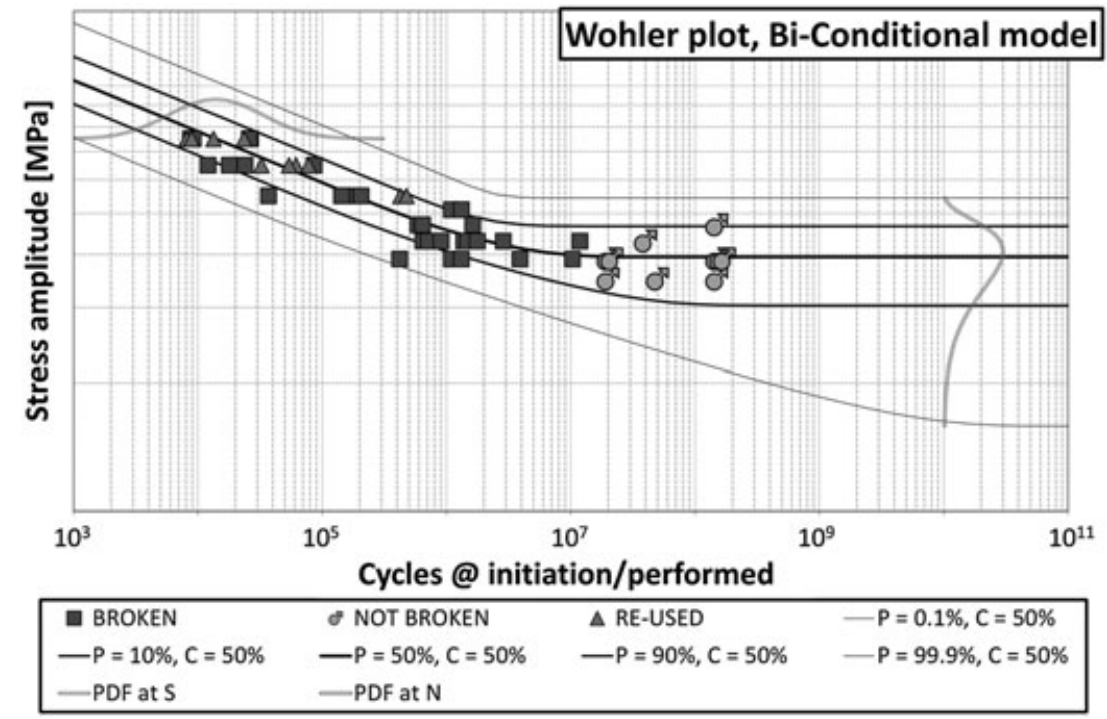

Fig. 5 Wohler plot of data and iso-probability curves according to the BCM. Here, a normal distribution has been used for $\mathrm{F}^{\text {end }}$ and a SEV for $\mathrm{F}^{\text {exi }}$. As a result, the marginal PDF of $\mathrm{N}$ at given $\mathrm{S}$ is a lognormal in the $\mathrm{F}^{\text {end }}$ regime and the marginal PDF of $\mathrm{S}$ at given $\mathrm{N}$ is a Weibull in the $\mathrm{F}^{\mathrm{exi}}$ area (as shown). 


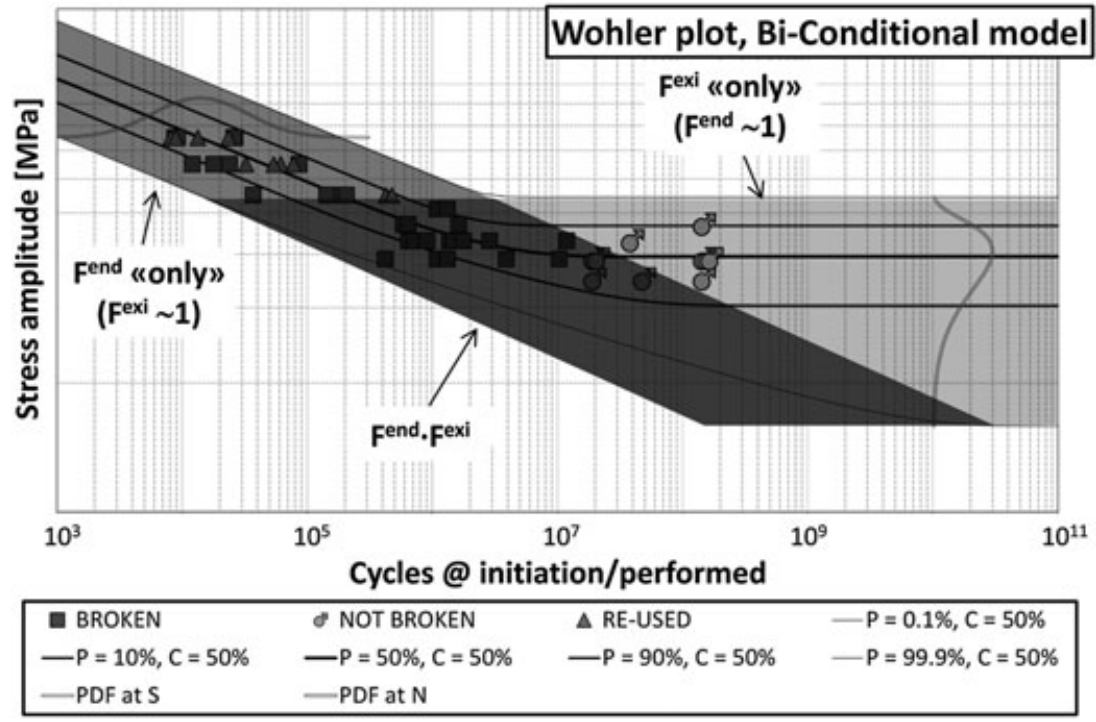

Fig. 6 Areas of influence of $\mathrm{F}^{\mathrm{end}}$ and $\mathrm{F}^{\mathrm{exi}}$.

\section{First estimate of the $F^{e x i}$ parameters: $\mu_{f}, \sigma_{f}$}

In estimating the parameters of the fatigue limit distribution, we need to consider also the unbroken, or run-out, specimens. As it has been suggested for the analysis of stair-case data, ${ }^{8}$ the parameters can be found by maximising a $\log$-likelihood of the $F^{x x i}$ in which

- all data are considered as right-censored (i.e. quantals); and

- the likelihood uses the CDF for the broken and the complimentary CDF for the run-outs. That is

$L\left(\boldsymbol{\theta}_{e x i}\right)=\prod_{i=1}^{n}\left[F^{e x i}\left(\bar{S}_{i}, \boldsymbol{\theta}_{e x i}\right)\right]^{\delta_{i}} \cdot\left[1-F^{e x i}\left(\bar{S}_{i}, \boldsymbol{\theta}_{e x i}\right)\right]^{1-\delta_{i}}$

where $\delta_{i}=1$ if the $\mathrm{i}$-th specimen is a failure, $\delta_{i}=0$ if is a not broken (run-out).

More practically, the log-likelihood to be maximised is

$$
\begin{aligned}
\mathscr{L}\left(\boldsymbol{\theta}_{e x i}\right) & =\ln \left[L\left(\boldsymbol{\theta}_{\text {exi }}\right)\right] \\
& =\sum_{i=1}^{n} \delta_{i} \ln \left[F^{e x i}\left(\bar{S}_{i}, \boldsymbol{\theta}_{e x i}\right)\right] \cdot\left(1-\delta_{i}\right) \ln \left[1-F^{e x i}\left(\bar{S}_{i}, \boldsymbol{\theta}_{e x i}\right)\right]
\end{aligned}
$$

It is important to notice how this formulation reveals the statistical weakness of the stair case approach, as discussed in the introduction. The quantal approach does not make use of valuable information such as the cycles to failure, and hence, the confidence interval of the estimated parameter is expected to be wider.

\section{Final estimate of all the parameters}

The first estimates can now be used as starting point for a maximisation of the likelihood of the $F^{B C}$, similarly to what has been suggested by Nelson ${ }^{22}$ :

$L(\boldsymbol{\theta})=\prod_{i=1}^{n}\left[f_{\overline{N_{f}}}\left(\bar{N}_{i}, \bar{S}_{i}, \boldsymbol{\theta}\right)\right]^{\delta_{i}} \cdot\left[1-F_{\overline{N_{f}}}\left(\overline{N_{i}}, \bar{S}_{i}, \boldsymbol{\theta}\right)\right]^{1-\delta_{i}}$

Again, it is easier to work with the log-likelihood, that is

$$
\begin{aligned}
\mathscr{L}(\boldsymbol{\theta})= & \sum_{i=1}^{n} \delta_{i} \ln \left[f_{\overline{N_{f}}}\left(\bar{N}_{i}, \bar{S}_{i}, \boldsymbol{\theta}\right)\right] \\
& +\left(1-\delta_{i}\right) \ln \left[1-F_{\overline{N_{f}}}\left(\bar{N}_{i}, \bar{S}_{i}, \boldsymbol{\theta}\right)\right]
\end{aligned}
$$

By comparing (22) with (20), the strengths of a P-S-N model with respect to the stair-case-like procedure may be appreciated: the quantal approach is used only where appropriate (i.e. the censored data) and the information of the cycles performed by each specimen is explicitly taken into account.

\section{Confidence intervals estimate}

Especially when dealing with low probabilities, the (maximum likelihood) estimate of the parameters and of the quantiles alone cannot be sufficient from the reliability point of view. Being able to take into account also for the finitude of the experimental data is crucial, and hence, it is desirable to have an explicit formula to lower the estimate as a function of the number of data points and desired confidence level. Unfortunately, this is possible 
only in some limited situations. ${ }^{23,24}$ In the following, two procedures are presented for the estimate of the confidence intervals for the parameters and the quantiles.

Confidence intervals for the estimate of the parameters

Confidence intervals for the parameters can be estimated using a likelihood ratio approach, in accordance to the field literature. ${ }^{13,21}$ If $\boldsymbol{\theta}=\left(\boldsymbol{\theta}_{1}, \boldsymbol{\theta}_{2}\right)$ is a partition of the vector of parameters $\boldsymbol{\theta}, \boldsymbol{\theta}_{1}$ is a vector of $k$ parameters of interest, and $\boldsymbol{\theta}$ is the ML estimate of $\boldsymbol{\theta},{ }^{13}$ the profile likelihood of $\boldsymbol{\theta}_{1}$ is defined as

$R\left(\boldsymbol{\theta}_{1}\right)=\max _{\theta_{2}}\left[\frac{\mathscr{L}\left(\boldsymbol{\theta}_{1}, \boldsymbol{\theta}_{2}\right)}{\mathscr{L}(\boldsymbol{\theta})}\right]$

If $k=1$, plotting $R\left(\boldsymbol{\theta}_{1}\right)$ results in a curve with an inverse U shape.

When evaluated at the true value $\boldsymbol{\theta}_{1}$, the asymptotic distribution of $-2 \log \left[R\left(\theta_{1}\right)\right]$ is a chi-squared distribution of with $k$ dof. ${ }^{25}$ It follows that an approximate 100 $(1-\alpha) \%$ confidence region for $\boldsymbol{\theta}_{1}$ is given by the set of $\boldsymbol{\theta}_{1}$ such that

$-2 \log \left[R\left(\boldsymbol{\theta}_{1}\right)\right] \leq \chi_{(k, 1-\alpha)}^{2}$

where $\chi_{(k, 1-\alpha)}^{2}$ is the $1-\alpha$ quantile of a chi-squared distribution with $k$ dofs. If $k=1$ (24) allows to compute the approximate confidence intervals for $\boldsymbol{\theta}_{1}$. Figure 7 shows the graphical representation of the profile likelihood approach.

\section{Confidence interval for the quantiles}

The procedure used for the parameters can be used also for the quantiles if one of the parameters can be explicitly expressed as a function of the other parameters and the quantile itself. ${ }^{25}$ This can be carried out using the formulation of the quantile at given S [Eq. (15)].

An effective alternative, although computationally more expensive, is the bootstrap method, whose application in this case is described in the following.

The basic idea is to draw a sample from the estimated distribution and then to fit the model on it. For each virtual case (specimen), a value of probability is chosen randomly, and by inverting the model CDF, a corresponding value of the variable(s) is derived. The set of virtual data can now be analysed the same way it has been carried out for the physical test. By doing so a large number of times, one can build a database of parameters and of quantiles estimate. The $1-\alpha$ confidence interval can then be computed by ranking these datasets. ${ }^{26}$

The concept of virtually replicating the test many times also implies that the same conditions and context

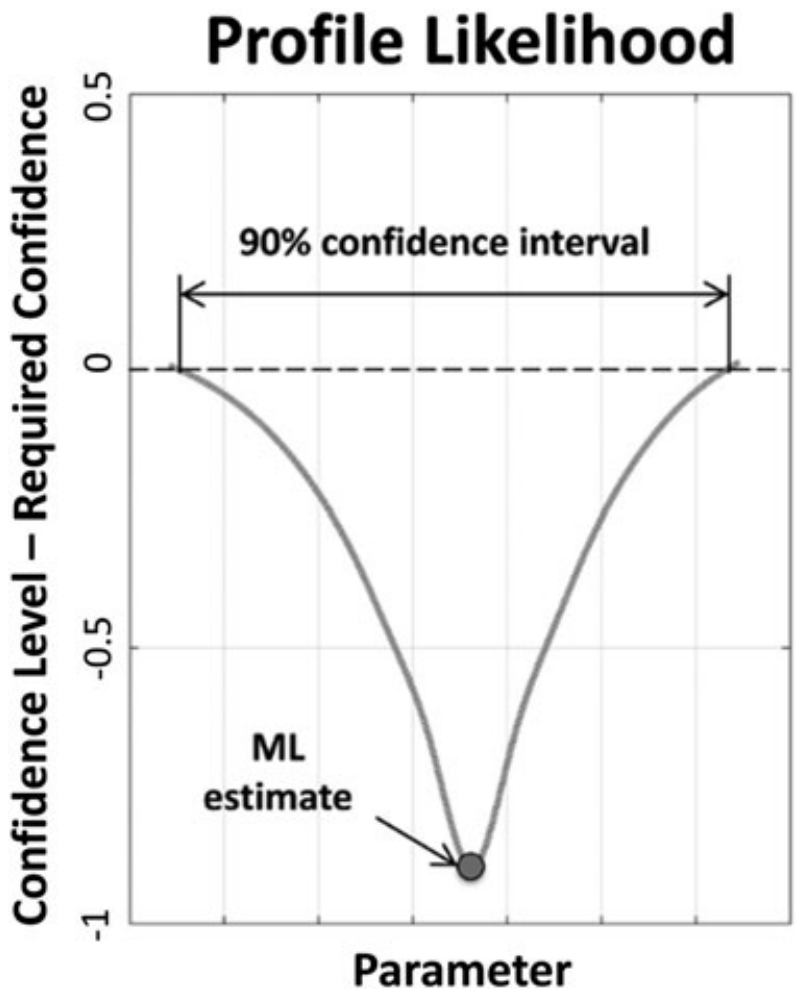

Fig. 7 Plot of $-2 \log \left[R\left(\theta_{1}\right)\right]-\chi_{(k, 1-\alpha)}^{2}$.

of the physical test should be pursued. One consequence is that the virtual samples cannot be chosen totally arbitrarily. In the following, the testing procedure is analysed.

In fatigue testing, the stress amplitude is the independent variable (the input value chosen by the user), and usually the test manager defines the stress levels basing on the ongoing behaviour of the test itself. If this procedure is structured, that is replicable and objectively defined, as in the case of the stair-case method, ${ }^{7}$ it can be virtually copied. If not, keeping the same stress amplitude set of the physical test is the most obvious choice. That is, we start by considering the set of $\bar{S}_{i}, i=1, \ldots, n$ of the log-stress amplitudes performed during the test.

For each $\mathrm{j}$-th loop, then, a vector of size $n$ (being $n$ the number of valid tests) of random values of probability $\mathrm{P}$ is drawn, named $\boldsymbol{P}_{j}$. For each couple $\bar{S}_{i}, P_{j, i}($ where $i=1, . ., n)$, we can find the corresponding number of cycles $\bar{N}_{j, i}^{v}$

$\bar{N}_{j, i}^{v}=\mathbb{Q}\left(\bar{S}_{i}, P_{j, i}\right)$

(where $v$ stands for virtual). It must be noted that $\bar{N}_{j, i}^{v} \in[1,+i n f]$, where $\bar{N}_{j, i}^{v}=$ inf when

$\bar{S}_{i}<\mathbb{Q}^{e x i}\left(P_{j, i}\right)$

or, in other words, when the applied stress is lower than the fatigue limit at the (randomly) chosen probability (15). 
In case of applications where censored data are involved, a further effort must be employed in order to properly take them into account.

For 'low' values of $\bar{N}_{j, i}^{v}$, no problem arises, but for 'high' number of cycles, we need to define whether the i-th specimen needs to be considered broken or runout. Even in the latter case, also, we must define the number of cycles performed in the virtual test. Again, with the aim of correctly replicate the physical test, we need to follow its same procedure.

In some cases, the run-out limit is pre-defined and is kept constant through the whole test. This is a simple scenario (Fig. 8a). In real applications, the situation can be very different, because of convenience mainly, and in some cases, it can happen to have specimens broken at a number of cycles actually higher than some censored (run-out) samples (Fig. 8b \& c).

To deal with this (general) context, we used and suggest the following. First, an array of all the $N_{i}$ from the real test greater or equal of the minimum run out is collected:

$\widetilde{N}=\left[\bar{N}_{i}>\min \bar{N}_{i}^{R-O}\right]$

where $N_{i}^{R-O}$ are the endurances of the run-out tests. This array can be seen as the availability (or 'willingness') of the laboratory to perform long runs. $\tilde{N}$ will then contain all the endurances of the run outs, plus some potential breaks.

In deriving the virtual endurances as described previously, each time the $\bar{N}_{j, i}^{v}$ is greater than $\min N_{i}^{R-O}$, we loop into $\tilde{N}$ to obtain the maximum number of cycles that the lab is willing to perform for that specimen.

If $\bar{N}_{j, i}^{v}<\widetilde{N}_{i}$, then $i$ is broken with $\bar{N}_{i}=\bar{N}_{j, i}^{v}$; Alternatively, if $\bar{N}_{j, i}^{v} \geq \widetilde{N}_{i}$, meaning the specimen do break, but the lab is not willing to let the test run that long, $i$ is a run-out with $\bar{N}_{i}=\widetilde{N}_{i}$.

At the end of this process, for each $j-t h$ bootstrap loop, we derive a virtual test outcome $\left[\overline{\boldsymbol{S}}, \overline{\boldsymbol{N}}^{v}, \boldsymbol{\delta}^{v}\right]_{j}$. The whole procedure is schematically shown in Fig. 9.

By fitting the model to the virtual data, with the aforementioned procedure, we then derive a set of parameters $\boldsymbol{\theta}_{j}$. With this, it is possible to numerically calculate the Pquantile at $S$ given $\mathrm{N}$ or in $\mathrm{N}$ given $\mathrm{S}$. In this paper, the bootstrapping procedure has been repeated 500 times for each fatigue test set.

Once the $\boldsymbol{\theta}_{j}$ and/or the quantiles are organised in ascending order, the $1-\alpha$ confidence interval is bounded by the values with rank $\frac{\alpha}{2}$ and $1-\frac{\alpha}{2}$. The quantile curves are then drawn by points, and the resulting plot is shown in Fig. 10.
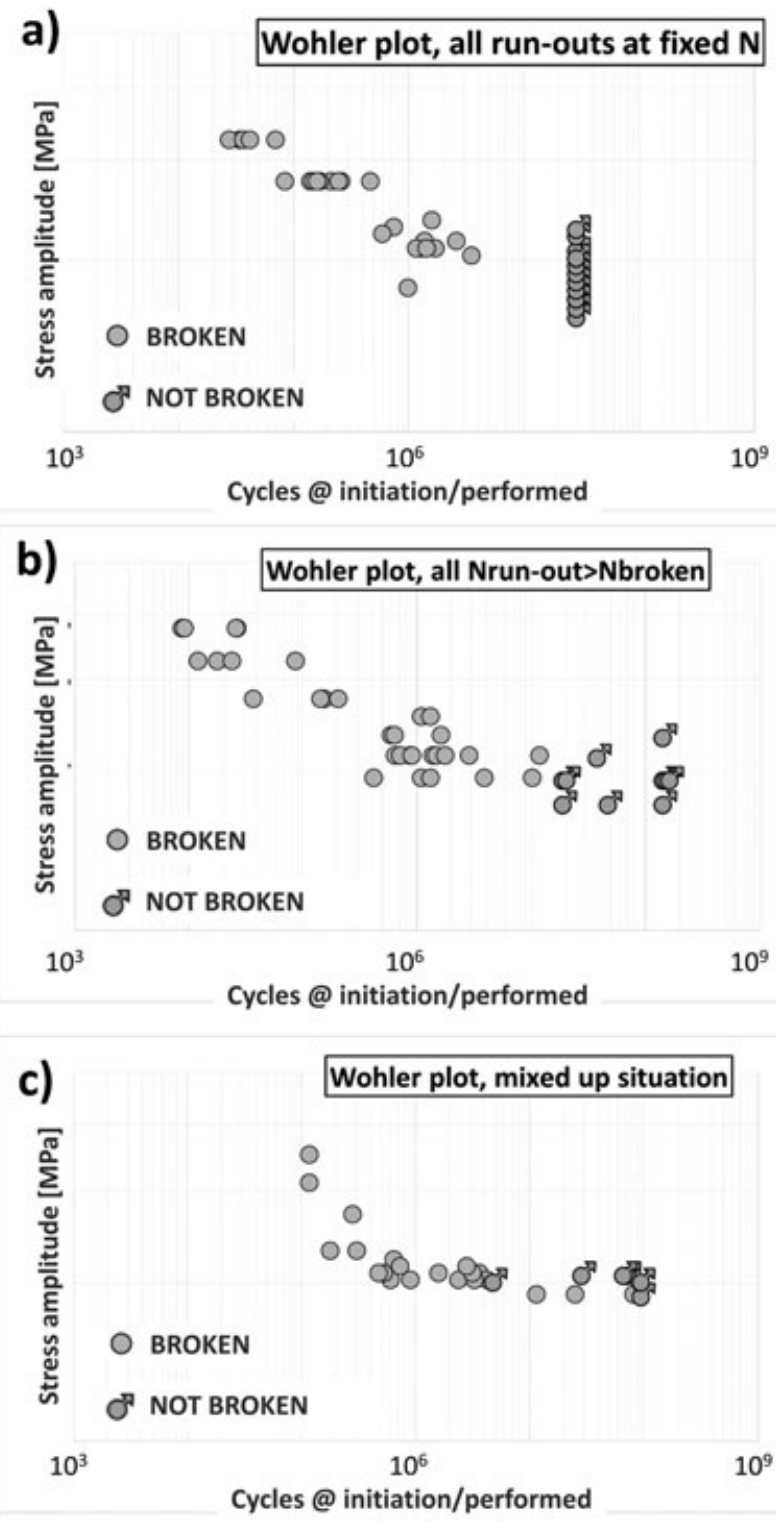

Fig. 8 (a) Fatigue test data where the run-out limit has been kept constant through the whole test; (b) Fatigue test data with variable run-out limit. In this case, however, it is still possible to define a number of cycles that divides the broken from the run-outs; (c) Fatigue test data in a more general configuration: the run out limit has not been kept constant, and some specimens broke at $\mathrm{N}$ higher than some run-outs.

In Table 1, the bootstrap estimates of the confidence interval of the parameters are reported and compared with those obtained with the relative likelihood method; results are referred to the dataset in Fig. 10. The two methods are in excellent agreement except for the scale parameter of $F^{e x i}$, which is actually the one that show the largest relative confidence interval.

In the author's knowledge, such a procedure has not been formalised yet and may be a valuable framework, which allows great freedom in test management. 


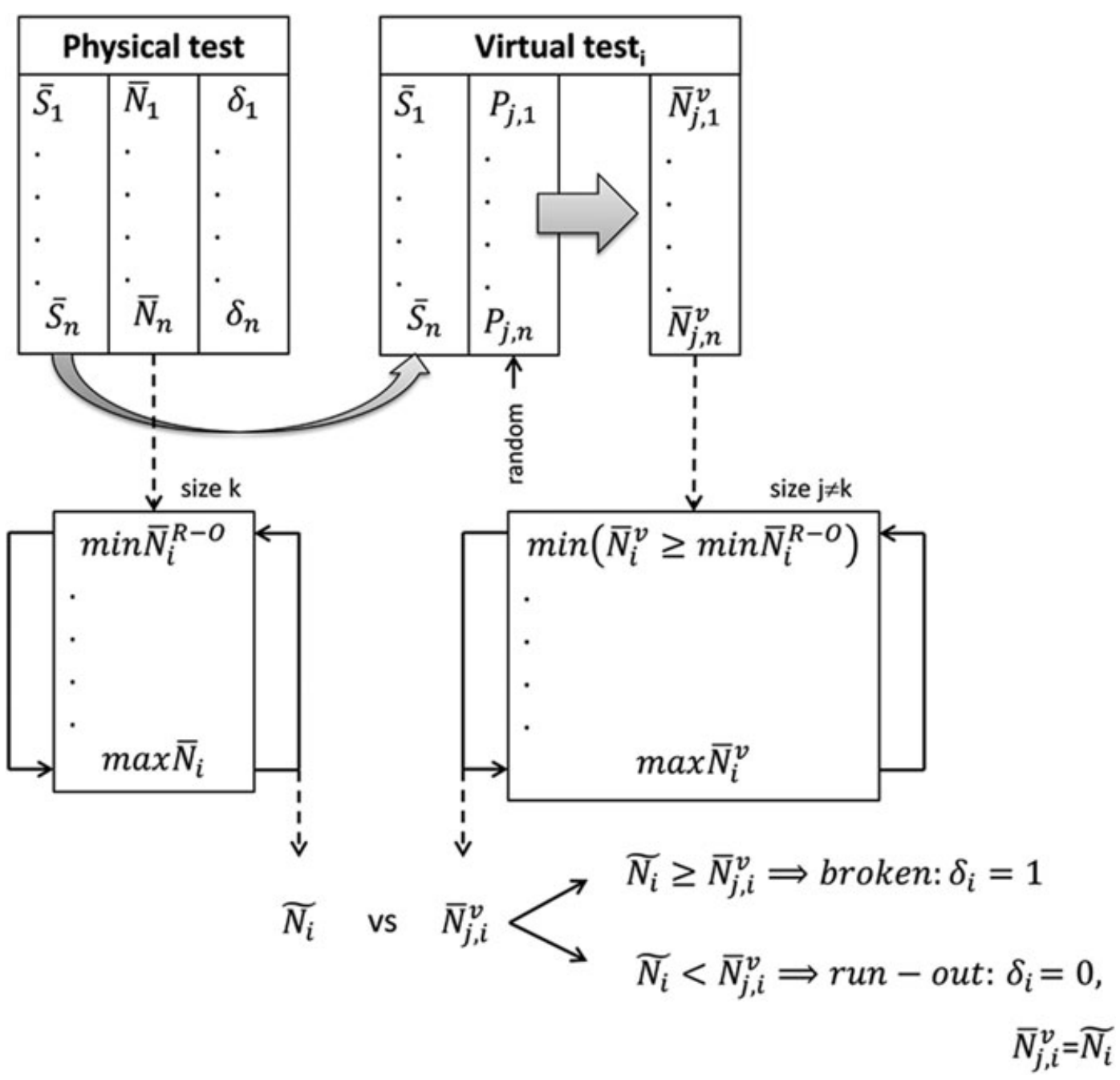

Fig. 9 Schematic representation of the virtual testing for the bootstrap procedure. Particular attention is focused on the definition of the run outs.

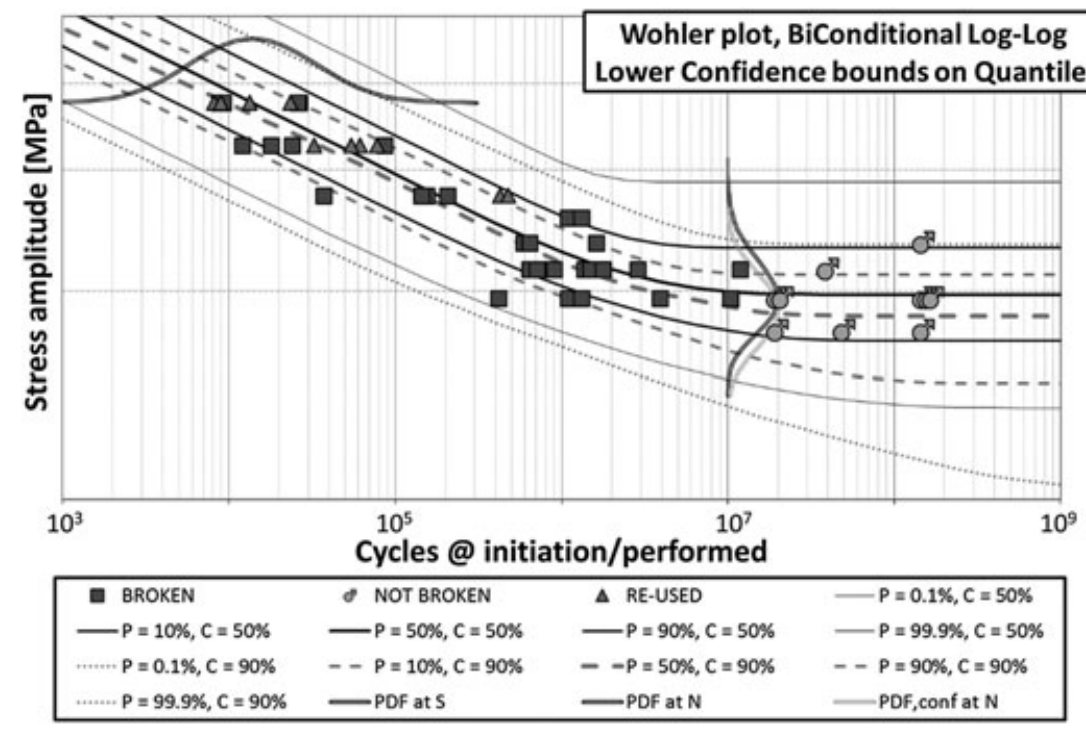

Fig. 10 Wohler plot with median and 90\% confidence lower bounds for some relevant quantile curves. Resulting confidence margin is larger for $F^{e x i}$ because of its inherent lower significance of the data. 
Table 1 Example of comparison between confidence bounds on parameters as calculated by likelihood ratio and bootstrap procedure with 500 iterations

\begin{tabular}{|c|c|c|c|c|c|}
\hline & $\begin{array}{l}\text { (Normalised) Maximum } \\
\text { likelihood estimate of } \\
\text { the parameter }\end{array}$ & \multicolumn{2}{|c|}{$\begin{array}{l}90 \% \text { confidence interval, } \\
\text { profile likelihood }\end{array}$} & \multicolumn{2}{|c|}{$90 \%$ confidence interval, bootstrap } \\
\hline A & 1 & 0.86 & 1.21 & 0.86 & 1.20 \\
\hline$\sigma_{e}$ & 1 & 0.81 & 1.27 & 0.77 & 1.21 \\
\hline $\mathrm{b}$ & 1 & 0.88 & 1.12 & 0.89 & 1.11 \\
\hline$\mu_{f}$ & 1 & 0.98 & 1.01 & 0.98 & 1.01 \\
\hline$\sigma_{f}$ & 1 & 0.56 & 2.05 & 0.37 & 1.57 \\
\hline
\end{tabular}

\section{POSSIBLE EXTENSIONS}

In the following, some variations of the model formulated previously are presented. All of them imply a variation in the number of parameters. In this regard, although maybe obvious, it is worth remembering the pros and cons of this topic. Every addition of a parameter is usually in the direction of having a model that is more adherent either to the data or to some theoretical assumptions. The first obvious counter effects are the computational and mathematical burdening. The more subtle, secondary consequence is that the confidence intervals become wider. Because, reversely, the sample size is generally inversely proportional to the uncertainty of the estimate, it is common sense that larger data sets allow for mode complex models. Although some statistical tools may come in handy, in our understanding, there is no 'right' solution to this problem, and it is only by engineering judgement and experience that one can make the most appropriate choice for the specific problem.

\section{Considering three-parameters distributions}

In principle, there is no restriction in using a threeparameter normal or SEV distribution instead of the most common two-parameter formulation. Obviously, the number of parameters of the model will increase. Particularly for $F^{e x i}$, the use of a threshold parameter allows for a $P=0$ lower bound in fatigue limit. This seems engineering sound, because it is common sense that no specimen would ever break at stress amplitude close to zero. On the other hand, however, the price is paid in terms of higher confidence intervals. This is generally true in the sense that the larger the number of parameters, the larger the confidence intervals. In the case of $F^{e x i}$, in particular, the estimate of the threshold is made even harder because of the scarcity of data in that region.

Finally, for the three-p Weibull in particular (i.e. the SEV for the log-data), it is known ${ }^{27,28}$ that the threeparameter formulation is poorly fitted by means of Maximum Likelihood methods.

\section{Heteroscedasticity}

Another possible extension of the model may be adding a further parameter to take a potential heteroscedastic behaviour in the HCF region into account, similarly to what has been proposed by Loren. ${ }^{21}$ As a first step, a linearly (in a log-log diagram) increasing scatter in fatigue life as stress decreases is the most reasonable choice (Beretta et al. ${ }^{29}$ ).

\section{Account for a change in slope after the knee point}

One of the basic assumptions of the model was the existence of a fatigue limit associated to the existence of the 'critical' defect. As a result of the model formulation, the $p$ quantile $\mathrm{S}-\mathrm{N}$ curves gradually connect the Basquin line to a horizontal asymptote. A different scenario, relevant to a different class on materials/processes/loading conditions, is the one where the $\mathrm{HCF}$ or $\mathrm{VHCF}$ region still has some slope. In this case, the second statistical event can be attributed to a different damaging mechanism. Again, if these two events are considered as statistically independent, the main framework of the BCM can be bent to a formulation where there are basically two $F^{e n d}$. Besides the extra parameter, this has a considerable impact in the presented parameter estimate procedure. Ideally, if there are sufficient data, one can replicate the initial estimate by a least square regression of the broken specimen in and after the knee point. The initial estimate of the scatter parameter could be more conveniently drawn by reducing the points to an arbitrary number of cycles instead of a stress level, because of the (usually) lower slope.

\section{Constant scatter in the HCF and VHCF regions}

Although not fully consistent with the model hypothesis, it is possible to reduce the number of parameters by employing the same type of distribution (normal or SEV) for $F^{e n d}$ and $F^{e x i}$ and the same scale parameter $\sigma_{e}=\sigma_{f}$. This reduces the number of parameters to four and is especially suited in case of small sample sizes. 


\section{Transition to the static strength (tensile test results)}

It is known that in the Basquin's law, the constant A [Eq. (5)] should be such that the maximum stress in the fatigue cycle at $\mathrm{N}=1$ is equal to the static strength (in true stress). If this is not explicitly set as a constraint, it usually happens that the value obtained from the parameter estimate is different. Even if this hypothesis is verified, it would be of interest to take into account the static strength distribution, which is, in general, different from that of fatigue.

Within the same framework, it is possible to extend the proposed model to include the static strength in probabilistic terms. If the static strength is seen as a stochastic variable independent from the fatigue one and if it is sufficiently higher than the fatigue limit, that is

$$
\begin{aligned}
& \operatorname{Pr}\left(\overline{S_{f}} \leq \bar{S}\right) \approx 1 \text { for } \bar{S} \text { close to the static strength } \\
& \operatorname{Pr}\left(\overline{S_{u}} \leq \bar{S}\right) \approx 0 \text { for } \bar{S} \text { close to the fatigue limit }
\end{aligned}
$$

it can be added to the equivalent system as a block in series with that of fatigue. This is because failure will occur if static strength or fatigue strength is reached (Fig. 11).

From the series and parallel system theory,

$R_{S}=\prod_{i=1}^{n} R_{i} \Rightarrow F_{S}=1-\prod_{i=1}^{n}\left(1-F_{i}\right)$

where $R_{S}$ is the system reliability and $F_{S}$ is the system probability of failure.

The uncertainty of the static strength can be modelled similarly to the fatigue limit:

$$
\begin{aligned}
F_{\overline{S_{u}}}^{s t} & =\operatorname{Pr}\left(\overline{S_{u}} \leq \bar{S}\right)=F^{s t}\left(\bar{S}, \mu_{s t}, \sigma_{s t}\right)=F^{s t}\left(\bar{S}, \theta^{s t}\right) \\
& =\Phi\left(\frac{\bar{S}-\mu_{s t}}{\sigma_{s t}}\right)
\end{aligned}
$$

With the same notation, $\Phi$ is again either the standard or the SEV CDF, $S_{u}$ is the static strength, $\mu_{s t}$ is the location parameter and $\sigma_{s t}$ is the scale parameter of the static strength.

(28), (29) and (13) can now be combined, and the resulting failure $\mathrm{CDF}$ becomes as follows:

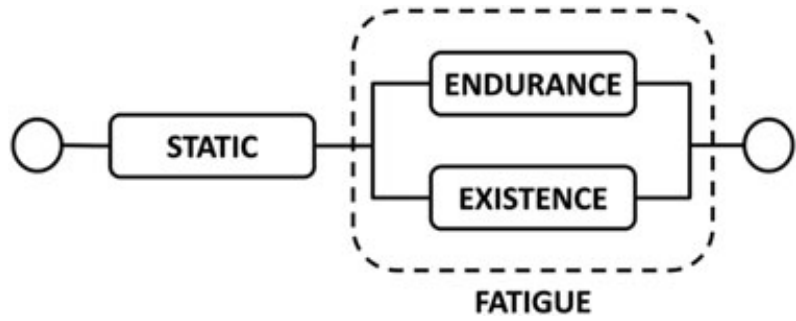

Fig. 11 Failure modelled as a serial/parallel system.

The marginal PDF is the partial derivative of $\mathrm{F}$ with respect to $\bar{N}$ :

$$
\begin{aligned}
& f_{\bar{N}}\left(\bar{N}, \bar{S}, \bar{A}, b, \sigma_{e}, \mu_{f}, \sigma_{f}, \mu_{s t}, \sigma_{s t}\right)=f_{\bar{N}}(\bar{N}, \bar{S}, \boldsymbol{\theta})=\frac{\partial F}{\partial \bar{N}}= \\
& =\frac{1}{b \sigma_{e}} \cdot \varphi\left(\frac{\bar{N}-b(\ln A-\bar{S})}{b \sigma_{e}}\right) \cdot \Phi\left(\frac{\bar{S}-\mu_{f}}{\sigma_{f}}\right) \cdot\left(1-\Phi\left(\frac{\bar{S}-\mu_{s t}}{\sigma_{s t}}\right)\right)
\end{aligned}
$$

where $\varphi$ is the PDF of the normal or the SEV.

The expression for the quantile in $\mathrm{N}$ at given $\mathrm{S}$ cannot be explicitly derived in the general form. However, making use of the hypothesis in (27), the quantile becomes as follows:

$\bar{N}(P, \bar{S})=\mathbb{Q}(P, \bar{S})$

$$
=\left\{\begin{array}{cc}
\mu_{e}(\bar{S})+\sigma_{e}{ }^{\prime} \Phi^{-1}\left(\frac{P-\Phi\left(\frac{\bar{S}-\mu_{s}}{\sigma_{s}}\right)}{1-\Phi\left(\frac{\bar{S}-\mu_{s}}{\sigma_{s}}\right)}\right) & \text { if } \bar{S}=\mu_{s}+\sigma_{s} \Phi^{-1}(P) \\
\mu_{e}(\bar{S})+\sigma_{e}^{\prime} \Phi^{-1}\left(\frac{P}{\Phi\left(\frac{\bar{S}-\mu_{f}}{\sigma_{f}}\right)}\right) & \text { for } \mu_{f} \bar{S} \cdot \mu_{s} \\
+\infty & \text { if } \bar{S}=\mu_{f}+\sigma_{f} \Phi^{-1}(P)
\end{array}\right.
$$

With the given formulation, the Wohler plot becomes like the one in Fig. 12.

$$
\begin{aligned}
F\left(\bar{N}, \bar{S}, \bar{A}, b, \sigma_{e}, \mu_{f}, \sigma_{f}, \mu_{s t}, \sigma_{s t}\right) & =F(\bar{N}, \bar{S}, \boldsymbol{\theta})=1-\left(1-\Phi\left(\frac{\bar{S}-\mu_{s t}}{\sigma_{s t}}\right)\right) \cdot\left(1-\Phi\left(\frac{\bar{N}-\mu_{e}(\bar{S})}{\sigma_{e}{ }^{\prime}}\right) \cdot \Phi\left(\frac{\bar{S}-\mu_{f}}{\sigma_{f}}\right)\right)= \\
& =\Phi\left(\frac{\bar{N}-\mu_{e}(\bar{S})}{\sigma_{e}{ }^{\prime}}\right) \cdot \Phi\left(\frac{\bar{S}-\mu_{f}}{\sigma_{f}}\right)+\Phi\left(\frac{\bar{S}-\mu_{s t}}{\sigma_{s t}}\right) \cdot\left(1-\Phi\left(\frac{\bar{N}-\mu_{e}(\bar{S})}{\sigma_{e}{ }^{\prime}}\right) \cdot \Phi\left(\frac{\bar{S}-\mu_{f}}{\sigma_{f}}\right)\right)
\end{aligned}
$$




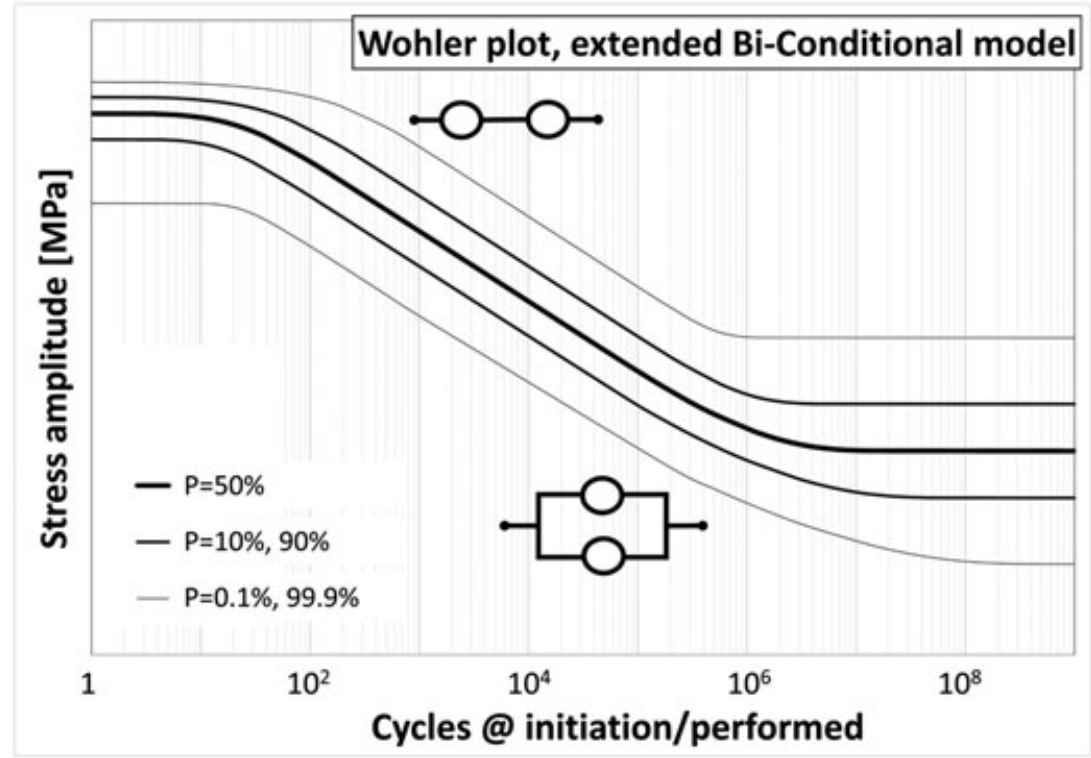

Fig. 12 Wohler plot at different failure probabilities. Here, the Weibull distribution has been used to model the static strength while $F^{\text {end }}$ and $F^{e x i}$ use a Lognormal.

As the parameter estimate and confidence interval are concerned, in principle, the aforementioned procedure can be followed, namely to separately estimate the parameters of the three distributions and then perform a final tuning with the full model (using an ML procedure). However, unless there are fatigue data in the Low Cycle Fatigue region (LCF, i.e. $N \in\left[10^{1} ; 10^{3}\right]$ ), in practice, the static and fatigue parameters can be estimated separately. In practice, the stress-life approach is actually not suitable for exploring the LCF, where an approach in deformation is more appropriate. The proposed extension does not claim to accurately and properly describe the LCF region. It is thus meant to be an alert on the probability of entering a different failure mode (the static strength), similarly to what has been proposed by Paolino et al. ${ }^{16}$ for the transition stresses between different Basquin lines. The concept of a series system applied to behaviour of materials is the mechanism of the 'competing risk model' presented by Harlow. ${ }^{18}$

\section{GENERALISATION: OTHER POSSIBLE USES}

The proposed model can actually be adapted to any physical phenomena or design schematization where there is a linear trend limited by threshold conditions and where it is reasonable to assume statistical independence of the underlying phenomena. Fatiguerelated examples are the Paris diagram and the Kitagawa diagram:

\section{Paris diagram (crack propagation)}

The $\mathrm{da} / \mathrm{dN}$ versus $\Delta K$ plot, in $\log -\log$ scale, has a central linear trend limited by a threshold for low values of $\Delta K\left(\Delta K_{t b}\right)$ and one for high values $\left(K_{I C}\right)$. Apart from the fact that the exponent is positive, that is the dependent variable is increasing with increasing of the independent one, the Paris diagram is indeed very similar to the Wohler plot as it is represented in Fig. 12, once the Wohler plot itself is correctly displayed with the independent variable (the $\Delta S$ ) in the abscissa. The Paris law is actually an exponential function such as the Basquin's. Experimentally, the three regions are smoothly connected, and some formulations ${ }^{30}$ have tried to explicitly describe this transition. But because the threshold $\Delta K_{t b}$, the linear relation $\mathrm{da} / \mathrm{dN}$ versus $\Delta K$ and the threshold $K_{I C}$ are also experimentally affected by some degree of scatter, they can be treated as stochastic variables, and under the same assumption as for the Wohler plot, the Paris diagram can be described by the present model. The non-propagating condition, that is there is no propagation if the applied $\Delta K$ is lower than the $\Delta K_{t h}$, and the Paris' law are a parallel system, in the sense that to have propagation, they both need to be satisfied. The unstable propagation condition, that is there is infinite increase in crack length for a single cycle, and the Paris' law are a serial system, because anyone of the two is sufficient for propagation. The formulation can be drawn by the one presented by substituting the fatigue limit with the threshold, the static strength with the $K_{I C}$, and the 
Basquin relation with the Paris law $\frac{d a}{d N}=C \Delta K^{n}$. The resulting marginal $\mathrm{CDF}$ is

$$
\begin{array}{r}
F\left(\frac{\overline{d a}}{\overline{d N}}, \overline{\Delta K}, \bar{C}, n, \sigma_{p}, \mu_{t b}, \sigma_{t b}, \mu_{I c}, \sigma_{I c}\right)= \\
=\Phi\left(\frac{\overline{\Delta K}-\mu_{I c}}{\sigma_{I c}}\right)+\Phi\left(\frac{\overline{\Delta K}-\mu_{t b}}{\sigma_{t b}}\right) \cdot \Phi\left(\frac{\frac{\overline{d a}}{d N}-\mu_{p}(\overline{\Delta K})}{\sigma_{p}}\right) \\
-\Phi\left(\frac{\overline{\Delta K}-\mu_{I c}}{\sigma_{I c}}\right) \cdot \Phi\left(\frac{\overline{\Delta K}-\mu_{t b}}{\sigma_{t b}}\right) \cdot \Phi\left(\frac{\frac{\overline{d a}}{d N}-\mu_{p}(\Delta K)}{\sigma_{p}}\right)
\end{array}
$$

where the pedix th refers to the distribution of $\Delta K_{t b}, I c$ to that of $K_{I C}, p$ to the Paris-Erdogan relation and
$\mu_{p}(\Delta K)=\bar{C}+n \overline{\Delta K}$. Figure 13a shows an example of a Paris plot reinterpreted by the present model. A similar model has been recently proposed by Paolino et al. ${ }^{31}$

\section{Kitagawa-Takahashi diagram}

As also highlighted by Morel et al., ${ }^{32}$ the strength because of a defect (crack) and that of the 'parent' defect-free material are independent probabilistic events that can be seen as a series system. In fact, for the material to fail, it is sufficient that just one of the two is satisfied. Moreover, in the extension proposed by Atzori and Lazzarin, ${ }^{33}$ another threshold is due to the condition of blunt notch. In this case, both the conditions of a $\Delta S$ greater than the blunt notch strength and the combination of $\Delta S$ and defect size must be satisfied in order for failure to occur

\section{a)}

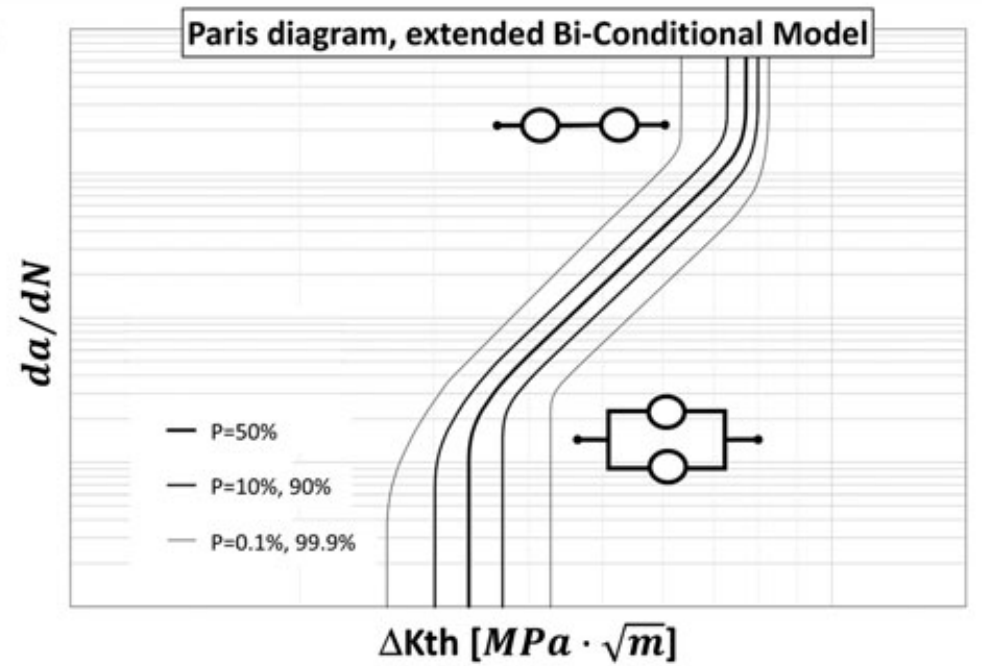

b)

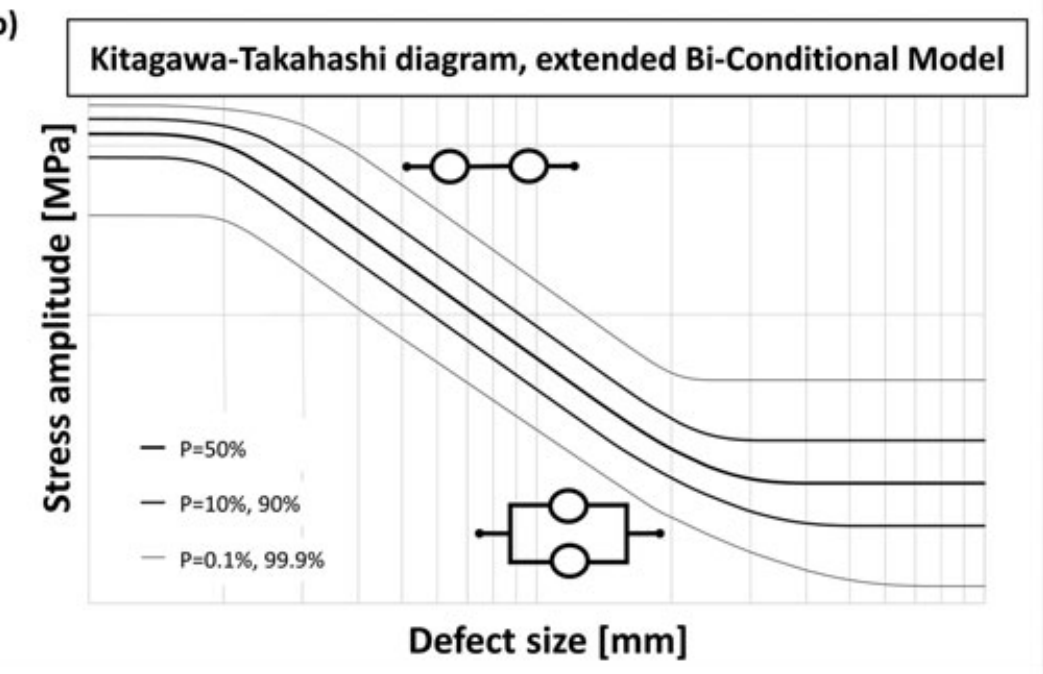

Fig. 13 (a) The Paris diagram reinterpreted using the extended Bi-conditional Model; (b) The Kitagawa-Takahashi diagram modelled as a series-parallel system using the extended Bi-conditional Model. 
(parallel system). Considering $S \cdot a^{1 / m=K}$ as a relation between the fatigue strength and the defect size $a$ (in LEFM, $m=2$ and $K=\frac{\Delta K_{t b}}{2 Y \sqrt{\pi}}$ with $Y$ being the shape factor), the resulting model is

$$
\begin{aligned}
F\left(\bar{a}, \bar{S}, K, m, \sigma_{L}, \mu_{0}, \sigma_{0}, \mu_{N}, \sigma_{N}\right)= \\
=\Phi\left(\frac{\bar{S}-\mu_{0}}{\sigma_{0}}\right)+\Phi\left(\frac{\bar{S}-\mu_{N}}{\sigma_{N}}\right) \cdot \Phi\left(\frac{\bar{a}-\mu_{L}(\bar{S})}{\sigma_{L}}\right) \\
-\Phi\left(\frac{\bar{S}-\mu_{0}}{\sigma_{0}}\right) \cdot \Phi\left(\frac{\bar{S}-\mu_{N}}{\sigma_{N}}\right) \cdot \Phi\left(\frac{\bar{a}-\mu_{L}(\bar{S})}{\sigma_{L}}\right)
\end{aligned}
$$

where the pedix 0 refers to the distribution of the fatigue strength in 'defect free' conditions, $N$ to that of the (blunt) notched condition, $L$ to the LEFM-like relation and $\mu_{L}(\bar{S})=m(\bar{K}-\bar{S})$. Figure 13b shows an example of a Kitagawa-Takahashi diagram reinterpreted by the present model.

\section{CONCLUSIONS}

The classic modelling of the Wohler curve as a linear relation between $N$ and $\Delta S$ (in a $\log -\log$ plot) with a lower threshold (the fatigue limit) is here reinterpreted in a probabilistic fashion. By considering these two events as independent stochastic events that both needs to happen for failure to occur, the system can be modelled as a parallel system, and thus, the overall probability of failure is the product of the individual probabilities.

Following this assumption, the presented model allows using different distributions both in formulation (Lognormal, Weibull, two or three-parameter formulations) and in scatter of the two events. As a result, the strength distributions are different in the HCF and in the VHCF regions. Practically speaking, the two models will be coupled only in the transition region between these two domains where the transition between the finite and infinite life comes out smooth as a result of the probabilistic setting. However, this is also the region that has usually the highest density of experimental data.

Under this framework, the case in which a probabilistic Basquin is used to model the finite life region is here expressed in details and represents a good balance between flexibility, applicability and simplicity. The resulting five-parameter CDF has been fitted with a procedure that has proven both robust and fast once implemented in an automated algorithm.

The derivation of the confidence interval for the parameters and the quantiles is a further step forward in reliability-oriented analysis. In accordance with the recent literature, the suggested procedure makes use of the profile likelihood, which is basically just a matter of more calculation time, because the ML fitting has to be repeated multiple times in order to draw the profile. An alternative approach is also presented using a bootstrap method. The matter of how to define the run-outs in the synthetic replicas of the testing arises. This is not trivial unless some heavy restrictions are imposed in the testing strategy. In an effort to cover the most general case, we present an innovative and robust analysis procedure.

Finally, an extension that includes the static strength with its probability distribution is presented and allows giving an alert about the onset of a different damaging mode. More generally, this probabilistic framework can be empowered whenever dealing with a linear trend that starts or ends to a threshold condition. Examples of application to the Paris and Kitagawa-Takahashi diagrams are provided.

The present model inserts itself in a line of recent models (see Refs. throughout the text). Similarly to these, most of the concepts are not entirely new but combined in a package that aims to fit a class of materials and practical needing. A comprehensive set of statistical tools is provided with focus on reliability applications at low probabilities and high number of cycles. A further step would be to test this model on multiple data sets and to compare it with other existing models.

\section{REFERENCES}

1 Marines, I., Bin, X. and Bathias, C. (2003) An understanding of very high cycle fatigue of metals. Int. F. Fatigue, 25, 1101-1107. DOI:10.1016/S0142-1123(03)00147-6.

2 Gilmore, C. (2015) Materials science and engineering properties, Cengage Learning: Boston; ISBN-13: 9781111988616 / ISBN-10: 1111988617

3 Basquin, O. H. (1910) The exponential law of endurance tests. Proc, Am Soc Test Mater, ASTEA, 10, 625-630.

4 Sakai, T., Lian, B., Takeda, M., Shiozawa, K., Oguma, N., Ochi, Y., Nakajima, M. and Nakamura, T. (2010) Statistical duplex S-N characteristics of high carbon chromium bearing steel in rotating bending very high cycle regime. Int. 7. Fatigue, 32, 497-504. DOI:10.1016/j.ijfatigue.2009.08.001.

5 Kauffman, H. (1998) Zur schwingfesten Bemessung dickwandiger Bauteile aus GGG-40 unter Berücksichtigung giesstechnisch bedingter Gefügeungänzen. Fraunhofer-Institut für Betriebsfestigkeit -LBF.

6 Berger, C. and Kaiser, B. (2006) Results of very high cycle fatigue tests on helical compression springs. Int. F. Fatigue, 28, 1658-1663.

7 Dixon, W. J. (1965) The up-and-down method for small samples. 7. Am. Stat. Assoc., 60, 967-978.

8 Engler-Pinto, C., Lasecki, J., Frisch, R., DeJack, M., Allison, J. (2005) Statistical approaches applied to fatigue test data analysis. SAE Technical Paper 2005-01-0802. doi:10.4271/ 2005-01-0802. 
9 Pollak, R. D. (2005) Analysis of methods for determining the high cycle fatigue strength of a material with investigation of Ti-6Al-4V gigacycle fatigue behavior. PhD Thesis, Dept. of Air Force, Air University, Air Force Institute of Technology.

10 Grove, D. and Campean, F. (2007) A comparison of two methods of analyzing staircase fatigue test data. Qual. Reliab. Engng. Int., 24, 485-497. DOI:10.1002/qre.899.

11 Little, R. E. (1990) Optimal stress amplitude selection in estimating median fatigue limits using small samples. F. Test. Eval., 18, 115-122.

12 Wallin, K. (2011) Statistical uncertainty in the fatigue threshold staircase test method. Int. 7. of Fatigue, 33, 354-362. DOI:10.1016/j.ijfatigue.2010.09.013.

13 Pascual, F. G. and Meeker, W. Q. (1999) Estimating fatigue curves with the Random Fatigue Limit Model. Technometrics, 41, 277-302. DOI:10.1080/00401706.1999.10485925.

14 Castillo, E. and Fernandez-Canteli, A. (2001) A general regression model for lifetime evaluation and prediction. Int. F. Fract, 107, 117-137.

15 Loren, S. and Lundstrom, M. (2005) Modeling curved S-N curves. Fatig. Fract Eng. Mater Struct, 28, 437-443. DOI:10.1111/j.1460-2695.2005.00876.x.

16 Paolino, D. S., Chiandussi, G. and Rossetto, M. (2012) A unified statistical model for S-N fatigue curves: probabilistic definition. Fatig Fract Eng. Mater Struct., 36, 187-201. DOI:10.1111/ j.1460-2695.2012.01711.x.

17 Paolino, D. S. and Cavatorta, M. P. (2013) On the application of the stochastic approach in predicting fatigue reliability using Miner's damage rule. Fatig Fract Eng. Mater Struct., 37, 107-117. DOI:10.1111/ffe.12093.

18 Harlow, D. G. (2011) Statistical characterization of bimodal behavior. Acta Mater., 59, 5048-5053. DOI:10.1016/j. actamat.2011.04.062.

19 Beretta, S., Anderson, C. and Murakami, Y. (2006) Extreme value models for the assessment of steels containing multiple types of inclusion. Acta Mater., 54(8), 2277-2289. DOI:10.1016/j.actamat.2006.01.016.

20 Rice, R. C. (1985) Fatigue data analysis. ASTM Metals Handbook. Vol 8, 9th ed.
21 Loren, S. (2003) Fatigue limit estimated using finite lives. Fatig. Fract Eng. Mater Struct, 26, 757-766.

22 Nelson, W. (1990) Accelerated testing: statistical models, test plans, and data analyses, John Wiley \& Sons: New York.

23 Owen, D. B. (1968) A survey of properties and applications of the noncentral t-distribution. Technometrics, 3(10), 445-478.

24 Shen C-L. (1994) The statistical analysis of fatigue data. PhD Thesis. Dept. of Aerospace and Mechanical Engineering, The University of Arizona.

25 Meeker, W. Q. and Escobar, L. A. (1998) Statistical methods for reliability data, John Wiley \& Sons: New York.

26 Efron, B. and Tibshirani, R. J. (1993) An introduction to the bootstrap, Chapman \& Hall: New York.

27 Heo, J.-H., Salas, J. D. and Kim, K.-D. (2001) Estimation of confidence intervals of quantiles for the Weibull distribution. Stoch Environ Res \& Risk Assess, 15(4), 284-309. DOI:10.1007/ s004770100071.

28 Cousineau, D. (2009) Fitting the three-parameter Weibull distribution: review and evaluation of existing and new methods. IEEE T Dielect El In, 16-1, 291-288. DOI:10.1109/ TDEI.2009.4784578.

29 Beretta, S., Clerici, P. and Matteazzi, S. (1995) The effect of sample size on the confidence of endurance fatigue tests. Fatig. Fract Eng. Mater Struct., 18, 129-139. DOI:10.1111/j.14602695.1995.tb00147.x.

30 "NASGRO® fracture mechanics and fatigue crack growth analysis software," Version 4.02, NASA-JSC and SwRI, September 2002.

31 Paolino, D. S. and Cavatorta, M. P. (2012) Sigmoidal crack growth rate curve: statistical modelling and applications. Fatig. Fract Eng. Mater Struct., 36, 316-326. DOI:10.1111/ffe.12001.

32 Pessard, E., Bellett, D. and Morel, F. (2013) A mechanistic approach to the Kitagawa-Takahashi diagram using a multiaxial probabilistic framework. Eng. Fract. Mech., 109, 89-104. DOI:10.1016/j.engfracmech.2013.06.001, <hal-00875771>.

33 Atzori, B., Lazzarin, P. and Meneghetti, G. (2003) Fracture mechanics and notch sensitivity. Fatig. Fract. Eng. Mater Struct, 26 , 256-267. 